\title{
"Caracterización clínico-epidemiológica de la enfermedad por coronavirus 2019 en edad pediátrica en San Pedro Sula, Honduras"
}

\author{
Risk factors for severe dengue mortality in pediatric patients at \\ Mario Catarino Rivas
}

\section{Norma Lidia Mejía Paz*, Néstor Raúl Banegas Mejía**, Nathalia Paredes Castillo**}

\section{RESUMEN}

Antecedentes: El 31 de diciembre de 2019, China comunicó un reporte de casos de neumonía de etiología desconocida. El 11 de febrero de 2020, la Organización Mundial de la Salud (OMS) denominó a la enfermedad por coronavirus 2019: COVID-19. ${ }^{(1)}$ En América Latina el primer caso se confirmó el 25 de febrero de 2020 en Brasil. (2) En Honduras se confirmaron los dos primeros casos de COVID-19 el 10 de marzo, ${ }^{(3)}$ y el primer caso en edad pediátrica en San Pedro Sula (S.P.S.) se confirmó el 25 de marzo de 2020. ${ }^{(4)}$ Objetivo: Caracterizar clínica y epidemiológicamente la enfermedad por coronavirus 2019 en la edad pediátrica en S.P.S., Honduras. Pacientes y métodos: Estudio cuantitativo, descriptivo, y transversal. Se utilizó un muestreo no probabilístico de 415 pacientes en edad pediátrica (IC 95\%) a quienes se les realizó una prueba de reacción en cadena de la polimerasa en tiempo real (PCR-tr). Las variables estudiadas fueron: sociodemográficas, curso de la enfermedad, manifestaciones clínicas, factores de riesgo, manejo del paciente, condición actual del paciente y distribución según el establecimiento de salud notificante. La información recolectada fue ingresada en una base de datos y analizada en Microsoft Excel. Resultados: 170 (40.96\%) pacientes en edad pediátrica resultaron positivos. El grupo etáreo más afectado fueron los adolescentes (54.71\%). El curso de la enfermedad fue sintomático en 124 pacientes. El síntoma que más frecuentemente se reportó fue fiebre $(37.10 \%)$.

\footnotetext{
*Epidemióloga Región Metropolitana de Salud de San Pedro Sula

**Médico Interno UNAH-VS

Dirigir correspondencia a: nestorbanegas40@gmail.com Recibido: 10 de Agosto de 2020 Aprobado: 20 de Septiembre de 2020
}

A la fecha de finalización del estudio, 152 continuaban activos con COVID-19. Conclusiones: Este estudio resume las principales características clínicas y epidemiológicas de la COVID-19 en niños y adolescentes de S.P.S.

\section{PALABRAS CLAVE \\ COVID-19, PCR, Pediatría}

\section{ABSTRACT}

Background: On December 31st, 2019 China released several case reports of unidentified etiology pneumonia. On February 11th, 2020, the World Health Organization (WHO) named the disease COVID-19 meaning coronavirus disease 2019. The first case was confirmed in Latin America on February 25th, 2020 in Brazil. In Honduras the first two cases of COVID-19 were confirmed on March 10th, 2020 and the first pediatric case in San Pedro Sula (S.P.S) was confirmed on March 25th, 2020. Objective: Characterize clinically and epidemiologically coronavirus disease 2019 in pediatric ages in San Pedro Sula, Honduras. Patients and Methods: Quantitative, descriptive and transversal study. We used a non probabilistic sample of 415 pediatric patients who underwent a real time polymerase chain reaction (PCR-tr) test. The studied variables were: sociodemographic, course of the disease, clinical manifestations, risk factors and patient management. The information gathered was submitted and analized in a Microsoft Excel database. Results: 170 (40.96\%) patients in pediatric age had a positive result. The most affected age group were teenagers $54.71 \%$ (93). The course of the disease showed that 124 patients were symptomatic. The most frequent clinical manifestation 
was fever (37.10\%). At the end of the study, 152 patients were still active with COVID-19. Conclusions: This study summarizes the clinical and epidemiological characteristics of COVID-19 in S.P.S.

\section{KEY WORDS}

COVID-19, PCR, Pediatrics

\section{INTRODUCCIÓN}

El 31 de diciembre de 2019, la Municipalidad de Wuhan en la Provincia de Hubei, República de China, comunicó un reporte de casos de neumonía de etiología desconocida. El 30 de enero de 2020, con más de 9,700 casos confirmados en China y 106 casos confirmados en 19 países, la Organización Mundial de la Salud (OMS) declaró el brote como una emergencia pública de preocupación internacional. El 11 de febrero, la OMS denominó a la enfermedad como COVID-19, que significaba enfermedad por coronavirus 2019. Esa misma fecha el Comité Internacional de Taxonomía de Virus anunció que el nuevo nombre del virus que causaba COVID-19 sería Coronavirus tipo 2 del Síndrome Respiratorio Agudo Severo (SARS-CoV-2). ${ }^{(1)}$ En América Latina el primer caso se confirmó el 25 de febrero de 2020 en Brasil. $^{(2)}$ En Honduras se confirmaron los dos primeros casos de COVID-19 el 10 de marzo, ${ }^{(3)}$ y el primer caso en edad pediátrica (de cero a dieciocho años) en San Pedro Sula (S.P.S.) se confirmó el 25 de marzo de 2020. ${ }^{(4)}$ Los coronavirus son virus pleomórficos de ARN monocatenario que miden entre 100 y $160 \mathrm{~nm}$ de diámetro. Su nombre deriva del aspecto similar a una corona que generan las proyecciones parecidas a bastos que se proyectan a partir de la cubierta viral. Los coronavirus que infectan a los seres humanos pertenecen a dos géneros: Alfacoronavirus y Betacoronavirus. El coronavirus del Síndrome Respiratorio Agudo Severo es un betacoronavirus. ${ }^{(5)}$ El coronavirus, un género de la familia Coronaviridae, del orden Nidovirales, ${ }^{(6)}$ infecta y altera el funcionamiento de las células epiteliales ciliadas, al ensamblarse al retículo endoplásmico rugoso en dos fases: 1) la fase inicial que produce una polimerasa de ARN (L) y 2) la fase tardía que produce proteínas estructurales y no estructurales a partir de un molde de ARN de sentido negativo. ${ }^{(7)} \mathrm{El}$ mecanismo patogénico que produce una neumonía es complejo. Los estudios recientes indican que la infección viral es capaz de producir una respuesta excesiva en el huésped. En algunos casos, ocurre una tormenta de citocinas, lo que resulta en un daño tisular extenso. La protagonista de la tormenta es la interleucina-6 (IL-6). ${ }^{(8)}$ El virus puede pasar a través de membranas mucosas, especialmente nasal y laríngea, luego entrar a los pulmones por el tracto respiratorio. El virus ataca los órganos diana que expresan la enzima convertidora de angiotensina 2 (ECA-2), como los pulmones, corazón, riñones y tracto gastrointestinal. El virus empieza un segundo ataque, causando que la condición del paciente se agrave en 7-14 días. ${ }^{\left({ }^{89}\right)}$ Los síntomas de un paciente infectado con SARS-CoV-2 varían desde mínimos síntomas a falla respiratoria severa con falla multiorgánica. ${ }^{(10)}$ En comparación con los adultos, el número de casos confirmados de COVID-19 en la edad pediátrica es bajo, y los índices de severidad y la tasa de mortalidad son incluso menores. ${ }^{(11)}$ Ésto se puede explicar a través de estudios en donde se observó una respuesta inmunológica mal adaptativa en los adultos, $\mathrm{y}$ mecanismos inmunológicos protectores en los niños. ${ }^{(12)}$ La presente investigación servirá para enmarcar los hechos y autores concretos que la pandemia produce en la población en edades pediátricas de nuestro municipio y derivará en la conducta oportuna que los Pediatras, el personal de salud asociado, las instituciones sanitarias públicas y privadas, los entes de enseñanza y las autoridades gubernamentales correspondientes deberán asumir de manera colegiada, científica, proactiva, decidida y oportuna. El objetivo de la investigación fue caracterizar clínica y epidemiológicamente la enfermedad por coronavirus 2019 en la edad pediátrica en S.P.S., Honduras.

\section{PACIENTES Y MÉTODOS}

Se realizó un estudio con enfoque cuantitativo, alcance descriptivo y de corte transversal sobre los pacientes en edad pediátrica (0-18 años) a los 
cuales se les tomó una muestra de reacción en cadena de la polimerasa en tiempo real (PCR-tr) para COVID-19 en S.P.S., Honduras, entre el 23 de marzo y el 23 de junio de 2020. La definición de caso sospechoso de COVID-19 fue: 1. Persona de cualquier edad que presentaba enfermedad respiratoria aguda con o sin antecedente de haber estado en contacto con un caso confirmado o sospechoso hasta 14 días antes del inicio de los síntomas, 2. Parte de un brote de casos, donde hay otros sospechosos o confirmados con infección por SARS-CoV-2, 3. Persona con cuadro clínico de insuficiencia respiratoria aguda inusitada sin una causa que explique el cuadro y, 4. Recién nacido de madre sospechosa o confirmada con infección por SARS-CoV-2, 14 días antes o 28 días después del nacimiento. ${ }^{(13)}$ Las muestras de PCR-tr se realizaron en la Red de Establecimientos de Salud que conformaron a la Región Metropolitana de S.P.S.: Hospitales Públicos, Hospitales Privados, Instituto Hondureño de Seguridad Social (IHSS) y Triajes de Atención COVID-19. Se utilizó un muestreo no probabilístico por conveniencia de 415 pacientes en edad pediátrica (IC 95\%). El criterio de inclusión fue: 1. Paciente en edad pediátrica con muestra de PCR-tr para COVID-19 entre el 23 de marzo y el 23 de junio de 2020, que residía en S.P.S., Honduras. Los criterios de exclusión fueron: 1 . Paciente en edad pediátrica con muestra de PCR-tr para COVID-19 entre el 23 de marzo y el 23 de junio de 2020, que no residía en S.P.S., Honduras. 2. Paciente mayor de 18 años con muestra de PCRtr para COVID-19. El método de recolección de datos fue la aplicación de la ficha de vigilancia epidemiológica de ETI-IRAG-COVID19. Luego de la aplicación del instrumento, los datos fueron ingresados y analizados en una base de datos en Microsoft Excel. Las variables estudiadas fueron: sociodemográficas, curso de la enfermedad, manifestaciones clínicas, factores de riesgo, manejo del paciente, condición actual del paciente y distribución según el establecimiento de salud notificador. Los resultados se presentaron como frecuencias, porcentajes, rangos y promedios de las variables estudiadas. La fuente de información para la recolección de datos fue secundaria, por lo que no se requirió utilizar consentimiento informado.

\section{RESULTADOS}

Entre el 23 de marzo y el 23 de junio de 2020 se realizaron 415 muestras de PCR-tr a pacientes en edad pediátrica en la Red de Establecimientos de Salud que conformaron a la Región Metropolitana de S.P.S., de las cuales 170 resultaron positivas (40.96\%) y 245 negativas (59.04\%). Las características sociodemográficas de los 170 pacientes con resultado positivo se presentaron en la

Tabla No. 1.

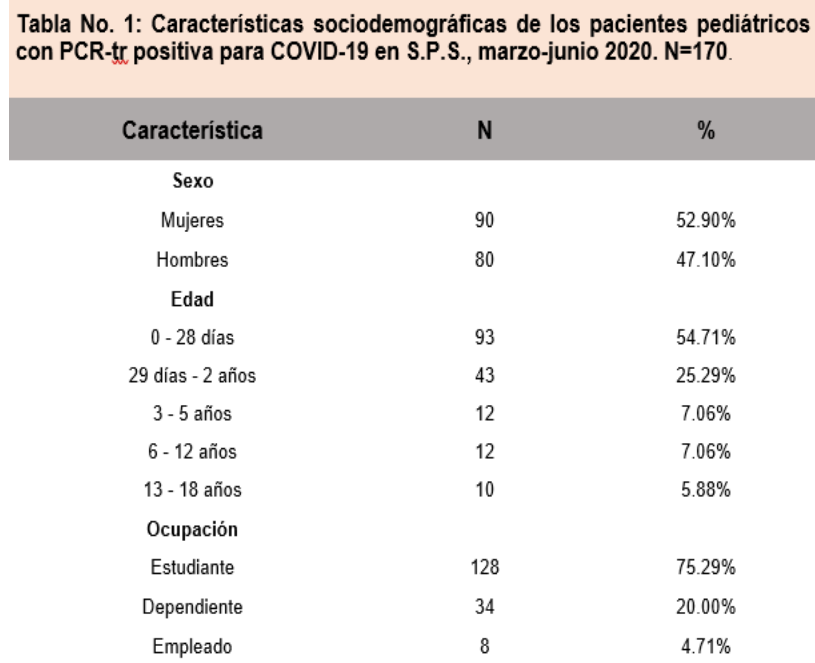

Fuente: Instrumento de investigación.

Los casos procedieron de 15 establecimientos de salud de la Región Metropolitana de Salud de S.P.S., de los cuales Miguel Paz Barahona (M.P.B.), Las Palmas y Fesitranh reportaron el mayor número de casos. (Ver Gráfico No.1) Gráfico No. 1: Procedencia por Establecimiento de Salud de los pacientes pediátricos con PCR-tr positiva para $\mathrm{CO}$ VID-19 en S.P.S., marzo-junio 2020. $\mathrm{N}=170$.

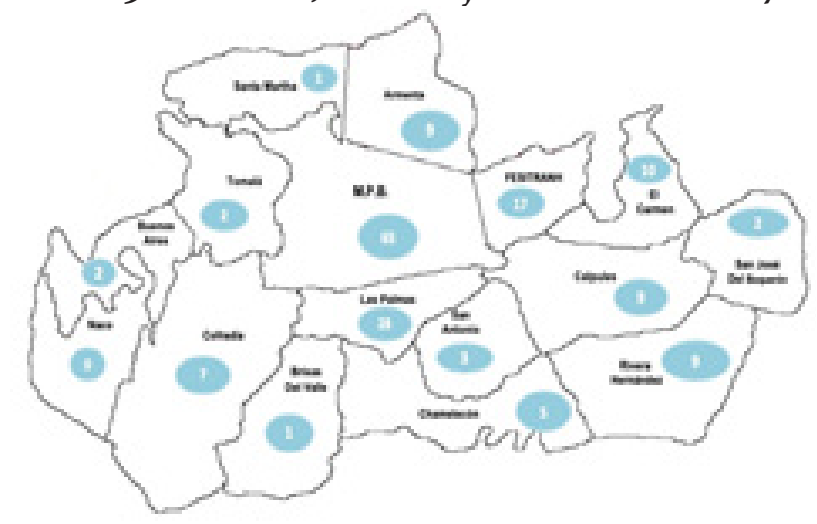


Fuente: Instrumento de investigación.

El curso de la enfermedad fue sintomático en 72.94\% (124) de los pacientes, y 27.06\% (46) estaban asintomáticos al momento de realizarse la PCR-tr.

De los 124 pacientes sintomáticos, el síntoma que se presentó con mas frecuencia fue fiebre en $27.06 \%$ (46) de los casos, seguido de cefalea en $20.59 \%$ (35) y tos en $19.41 \%$ (33).

(Ver Gráfico No. 2)

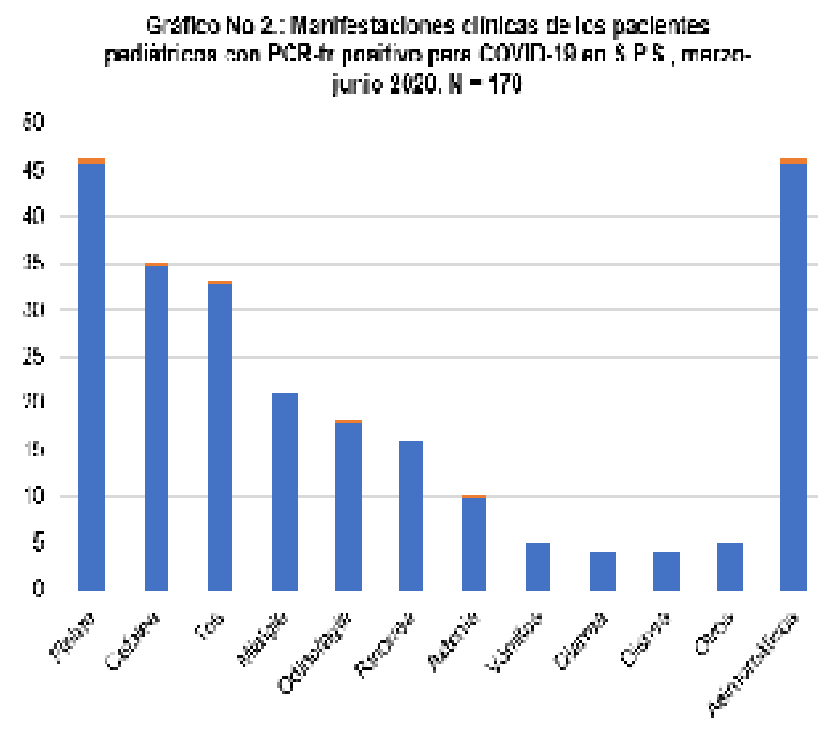

Fuente: Instrumento de investigación

En la distribución por factores de riesgo, 4.12\% (7) de los pacientes positivos tenían uno de los siguientes: embarazo, asma, diabetes tipo 1 o cardiopatía congénita. (Ver Gráfico No. 3)

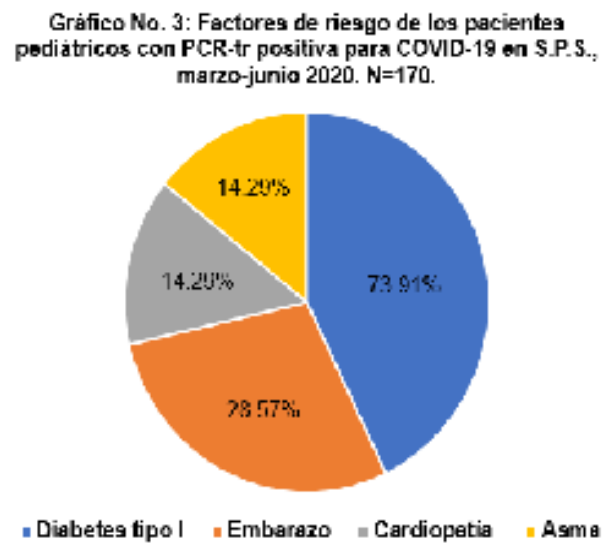

Fuente: Instrumento de investigación
El manejo del paciente fue en el 85.88\% (146) de los casos aislamiento domiciliario. $14.12 \%$ (24) de los pacientes requirieron aislamiento hospitalario.

A la fecha de finalización del estudio, $89.41 \%$ (152) pacientes continuaban activos con COVID-19, y $10.59 \%$ (18) se habían recuperado (según la primera prueba control).

De los establecimientos notificadores de casos positivos, las Unidades COVID-19 reportaron el 67.05\% (114) de los casos. (Ver Gráfico No. 4)
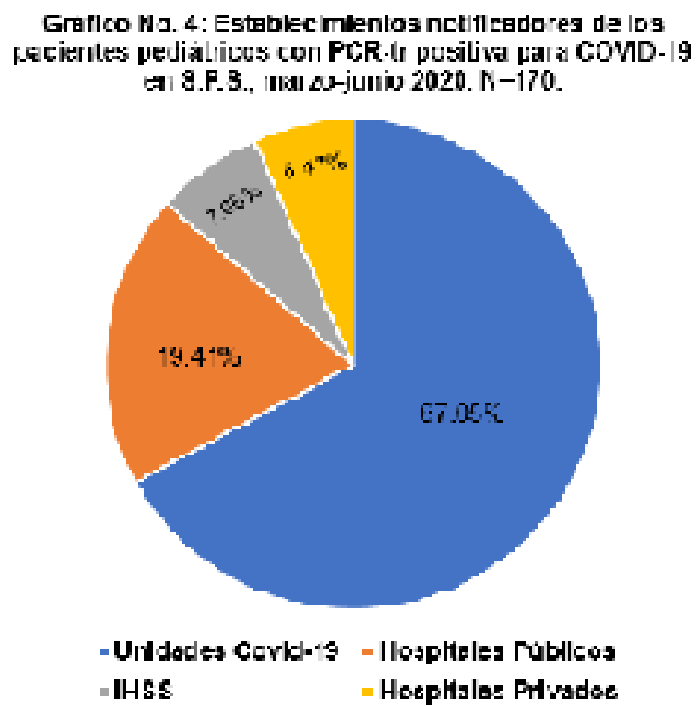

Fuente: Instrumento de investigación.

\section{DISCUSIÓN}

El SARS-CoV-2 es altamente infeccioso; la población entera es susceptible, y las gotas respiratorias y el contacto directo son las principales rutas de transmisión. ${ }^{(14)}$ La mayoría de los estudios han demostrado que el coronavirus ha afectado a la población pediátrica, y aunque la información es limitada, ya se han observado casos leves, moderados y severos en todos los grupos de edad. (15) Algunos estudios han sugerido que la mayoría de la población pediátrica infectada cursó con síntomas leves y tuvo mejor pronóstico que los adultos. ${ }^{(16)}$ En el análisis de la distribución por sexo de pacientes positivos por COVID-19, se encontró en un estudio randomizado llevado a cabo en el Karolinska Institutet en Estocolmo, Suecia $^{(17)}$ 
que de 2,143 pacientes pediátricos con COVID-19, 56.6\% fueron hombres. Asimismo, en el reporte del Centro de Diagnóstico de Enfermedades $(C D C)^{(18)}$ en abril de 2020 , se observó que de 2,490 pacientes pediátricos, $57 \%$ fueron hombres. Contrario a lo observado en nuestra investigación, en donde el 52.94\% fueron mujeres y $47.06 \%$ fueron hombres.

En una caracterización epidemiológica en niños realizada por Choi y cols en China, ${ }^{(19)}$ se observó que el $56.89 \%$ de los casos positivos fueron niños mayores de 10 años. $\mathrm{Al}$ analizar los datos epidemiológicos relacionados a la edad media de pacientes pediátricos con COVID-19 en un estudio transversal realizado por Shekerdemian et al en Norte América, ${ }^{(20)}$ se observó que de 48 pacientes, la edad media fue 13 años. El CDC ${ }^{(18)}$ reportó que dentro de los 2572 pacientes pediátricos con COVID-19, 32\% tenían entre 15-17 años, 27\% entre 10-14 años, $15 \%$ entre 5-9 años, $11 \%$ entre $1-4$ años y $15 \%$ eran pacientes menores de 1 año de edad. En un estudio de cohorte observacional realizado en tres hospitales de Zhejiang, China, entre el 17 de enero y el 1 de marzo del $2020^{(21)}$, se encontró que de 36 pacientes pediátricos positivos para COVID-19, 10 eran menores o igual a 5 años y 26 tenían entre 6 y 16 años. En nuestra investigación se observó que el $54.71 \%$ de los pacientes positivos tenían mas de 12 años.

(Ver Tabla No. 1)

En una revisión sistemática de 14 estudios realizada por Castagnoli et $\mathrm{al}^{(22)}$,), se encontró que hasta esa fecha se habían reportado casos pediátricos sintomáticos y asintomáticos de COVID-19. En otro estudio en Brasil, por de Souza y cols ${ }^{(23)}$, observaron que de 1,117 casos, $14.2 \%$ (159) fueron asintomáticos y $85.8 \%$ (958) fueron sintomáticos. En nuestro estudio encontramos que el $72.94 \%$ de los pacientes fueron sintomáticos y $27.06 \%$ fueron asintomáticos.

Según el estudio realizado por de Souza y cols(23), el síntoma mas frecuente fue la fiebre, reportado en el $47.5 \%$ de los casos. Otros síntomas fueron tos $(41.5 \%)$, rinorrea (11.2\%), dia- rrea $(8.1 \%)$, vómitos $(7.1 \%)$, fatiga (5\%) y distrés respiratorio (3.5\%). El síntoma mas frecuente en nuestro estudio fue la fiebre, seguido por cefalea y tos. (Ver Gráfico No. 2)

Al observar la distribución por factores de riesgo, se encontró en un estudio retrospectivo realizado en 10 hospitales públicos de Hubei, China por Zheng et $\mathrm{al}^{(24)}$ que de 25 pacientes pediátricos con COVID-19, dos tenían factores de riesgo; uno de ellos tenía una cardiopatía congénita, malnutrición y sospecha de enfermedad metabólica hereditaria, y el segundo paciente tenía una cardiopatía congénita. Asimismo, en una revision sistemática de 131 artículos en Texas, Estados Unidos $^{(25)}$ se encontró que 233 pacientes tenían factores de riesgo, siendo el mas frecuente la inmunosupresión (30.5\%), seguido de factores de riesgo respiratorios y cardiovasculares. En nuestro estudio se identificaron 7 pacientes positivos con factores de riesgo: embarazo, asma, diabetes tipo 1 o cardiopatía congénita.

(Ver Gráfico No 3.)

Este estudio resume las principales características clínicas y epidemiológicas de la COVID-19 en niños y adolescentes de San Pedro Sula. Se necesitarán mas investigaciones para identificar posibles estrategias preventivas y terapéuticas.

\section{CONFLICTOS DE INTERÉS}

Los autores declaran que no existe conflicto de interés en la publicación del presente artículo.

\section{REFERENCIAS BIBLIOGRÁFICAS}

1. Pan American Health Organization / World Health Organization. Epidemiological Update: Novel coronavirus (COVID-19). 28 February 2020, Washington, D.C.: PAHO/WHO; 2020.

2. Rodriguez-Morales AJ, Gallego V, Escalera-Antezana JP, Mendez CA, Zambrano LI, Franco-Paredes C, et al. COVID-19 in Latin America: The implications of the first confirmed case in Brazil. Travel Medicine and Infectious Disease. 2020. DOI: https://doi.org/10.1016/j. tmaid.2020.101613. 
3. Secretaría de Salud de Honduras. Salud confirma los dos primeros casos de COVID-19 en el territorio hondureño. 11 de marzo de 2020. Disponible en: https://covid19honduras.org/?q=primeros-casos-confirmados

4. Secretaría de Salud de Honduras. Comunicado \#14 con 16 nuevos casos llegando a 52 confirmados. Disponible en; https://covid19honduras. org/?q=comunicado-14

5. Dolin R. Infecciones por Coronavirus. Harrison: Principios de Medicina Interna. 19 ed. Estados Unidos: Mc Graw Hill. 2016:1203-1206.

6. Perlman S and Netland J. Coronaviruses postSARS: update on replication and pathogenesis. Nature Reviews (2009): 7(1); pp. 439-450. doi:10.1038/nrmicro2147

7. Murray P, Rosenthal K, Pfaller M. Microbiología Médica. 7 ed. Estados Unidos: Elsevier; 2014; pp 506-510.

8. Di Gennaro F, Pizzol D, Marotta C, Antunes M, Racalbuto V, Veronese N, Smith L. Int. J. Environ. Res. Public Health 2020, 17, 2690; doi:10.3390/ijerph17082690

9. Chen, C.; Zhang, X.R.; Ju, Z.Y.; He, W.F. Advances in the research of cytokine storm mechanism induced by Corona Virus Disease 2019 and the corresponding immunotherapies. Zhonghua Shao Shang Za Zhi 2020, 36. DOI: 10.3760/ cma.j.cn501120-20200224-00088

10. Yuki, K; Fujiogi M; Koutsogiannaki S. COVID-19 pathophysiology: A review. Clinical Immunology 215 2020. DOI: https://doi.org/10.1016/j.clim.2020.108427

11. Morand, A; Fabre A; Minodier P; Boutin A; Vanel N; Bosdure E; Fournier PE. COVID-19 virus and children: What do we know? Editorial / Archives de Pédiatre 27 (2020), pp. 117-118. DOI: https://doi.org/10.1016/j.arcped.2020.03.001
12. Dhochak N; Singhal T; Kabra SK; Lodha R. Pathophysiology of COVID-19: Why Children Fare Better than Adults? The Indian Journal of Pediatrics. May 2020. DOI: https://doi. org/10.1007/s12098-020-03322-y

13. Sociedad Hondureña de Enfermedades Infecciosas, Sociedad Hondureña de Cuidados Intensivos, Sociedad Hondureña de Alergia e Inmunología Clínica, Asociación Pediátrica Hondureña. Protocolo de Atención al Paciente Pediátrico con Sospecha o Infección confirmada por SARSCoV-2 (COVID-19). 2020. Disponible en: http:// www.salud.gob.hn/site/index.php/component/ edocman/protocolo-covid-pacientes-pediatricos

14. Hong H; Wang Y; Chung HT; Chen CJ. Clinical characteristics of novel coronavirus disease 2019 (COVID-19) in newborns, infants and children. Pediatrics and Neonatology (2020) 61, 131-132. DOI: https://doi.org/10.1016/j.pedneo.2020.03.001

15. Saleem H, Rahman J, Aslam N, Murtazaliev S, Khan S. Coronavirus Disease 2019 in Children: Vulnerable or Spared? A Systematic Review. Cureus. 2020, 12(5): e8207. DOI: 10.7759/ cureus.8207

16. Guan WJ, Ni ZY, Hu Y. Clinical characteristics of coronavirus disease 2019 in China. New England Journal of Medicine. 2020, 382:17081729. DOI: 10.1056/NEJMoa2002032

17. Ludvigsson JF. Systematic review of COVID-19 in children shows milder cases and a better prognosis than adults. Acta Paediatrica. 2020; 109:1088-1095. DOI: 10.1111/apa.15270

18. CDC COVID-19 Response Team. Coronavirus Disease 2019 in Children - United States, February 12 - April 2, 2020. 69:14:422-426.

19. Choi SH, Kim HW, Kang JM, Kim DH, Cho EY. Epidemiology and clinical features of coronavirus disease 2019 in children. Check for Updates. 2020; 63:4:125-132. DOI: https://doi. org/10.3345/cep.2020.00535 
20. Shekerdemian LS, Mahmood NR, Wolfe KK, Riggs BJ, Ross CE, Heidemann SM, et al. Characteristics and Outcomes of Children with Coronavirus Disease 2019 Infection Admitted to US and Canadian Pediatric Intensive Care Units. JAMA Pediatrics. 2020, e1-e6. DOI: 10.1001/jamapediatrics.2020.1948

21. Qiu H, Wu J, Hong L, Luo Y, Song Q, Chen D. Clinical and epidemiological features of 36 children with coronavirus disease 2019 in Zhejiang, China: an observational cohort study. The Lancet. 2020; 20:689-696. DOI: https://doi. org/10.1016/ S1473-3099(20)30198-5

22. Castagnoli R, Votto M; Licari A, Brambilla I, Bruno R, Perlini S et al. Severe Acute Respiratory Syndrome Coronavirus 2 (SARS-CoV-2) Infection in Children and Adolescents: A Systematic Review. JAMA Pediatrics. 2020. DOI: 10.1001/ jamapediatrics.2020.1467

23. Souza T, Nadal J, Nogueira R, Pereira R, Brandao M. Clinical Manifestations of Children with COVID-19: a Systematic Review. Pediatric Pulmonology. 2020. DOI: 10.1002/ppul.24885

24. Zheng F, Liao C, Fan Q, Zhao X, Xie Z, Li $\mathrm{X}$, et al. Clinical Characteristics of Children with Coronavirus Disease 2019 in Hubei, China. Current Medical Science. 2020; 40(2):275-280. DOI: https://doi.org/10.1007/s1 1596-020-2172-6

25. Hoang A, Chorath K, Moreira A, Evans M, Moreira A, Naqvi R, et al. COVID-19 in 7780 pediatric patients: A systematic review. E Clinical Medicine. 2020. DOI: https://doi.org/ 10.1016/j. eclinm.2020.100433 